\section{Grenzrauschen. Zur Figur des Parasiten in der Systemtheorie}

\section{Border noise: On the Figure of the Parasite in Systems Theory}

\section{Matthias Leanza}

\begin{abstract}
:
At the heart of systems theory (N. Luhmann) is the problem of closure. One of the key questions is: How does a system close itself by drawing a boundary between itself and the environment? The ways in which a system generates and maintains its boundary depends on the type of operation it performs. Against this background it is, at first, astonishing that since the mid-1980s Luhmann has repeatedly referred to Michel Serres' book Le parasite. According to Serres, the figure of the parasite symbolises the impossibility of operational closure and stable boundaries. It is the excluded that returns to the inside. It is in this respect, this article argues, that the figure of the parasite cannot be incorporated into systems theory without rethinking operational closure and systemic boundaries. After having elaborated this problem, some of the main aspects of Serres' figure of the parasite will be reconstructed in order to adapt them within systems theory. It will be suggested to conceive of the parasite as an operation that confirms and rejects the operational closure of its host system. It creates order and disorder at the same time. Furthermore this paper argues that the notion of the parasite allows a more comprehensive understanding of modern society - an understanding that overcomes the temptation to behold a clear-cut order everywhere.
\end{abstract}

Matthias Leanza is a research assistant at the Department of Sociology, University of Freiburg. Among his research interests are systems theory, poststructuralist theories, theory of society. Email: matthias.leanza@soziologie.uni-freiburg.de.

\section{Keywords:}

dt.: Grenze, operative Schließung, Differenzierung, Entdifferenzierung, Michel Serres

engl.: boundary, operational closure, differentiation, dedifferentiation, Michel Serres

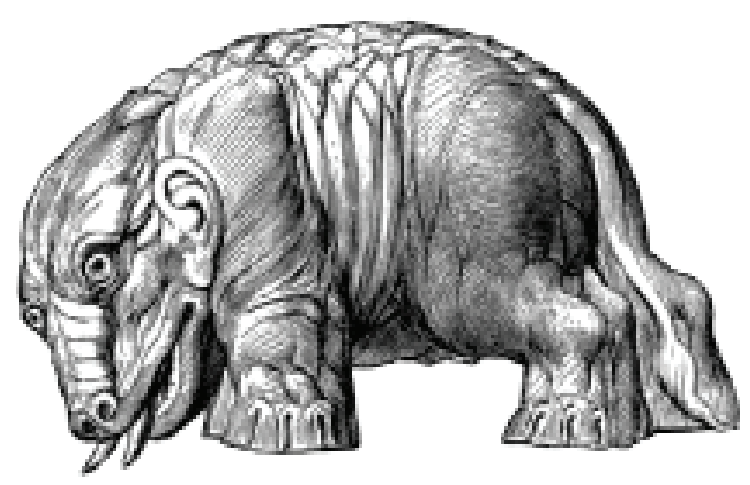




\section{Der Parasit zwischen System und Umwelt}

„Wie ist soziale Ordnung möglich?“ - einer verbreiteten Auffassung zufolge steht diese Frage im Zentrum der Soziologie. Niklas Luhmann $(1993,195)$ bezeichnete sie gar als eine für „die Disziplin konstituierende Problemstellung“. Und in der Tat hat jede Sozialtheorie zu klären, wie soziale Ordnungsbildung möglich ist und in welchen Formen sie sich vollzieht. Freilich existieren soziologische Einzelforschungen, die nicht explizit auf diese Frage reflektieren; dennoch müssen auch sie den Bezug zu den im Fach etablierten Vorstellungen von Sozialität wahren, möchten sie als ein Beitrag zum soziologischen Erkenntnisgewinn verstanden werden. Im Umkehrschluss bedeutet das: Vermag eine Theorie nicht anzugeben, wie soziale Ordnung entsteht, kann sie auch nicht an der für die Disziplin prägenden Problemperspektive partizipieren. Sie bleibt dann soziologisch undiszipliniert und muss mit einem Platz außerhalb des Fachs vorlieb nehmen.

Im Mittelpunkt der systemtheoretischen Ordnungskonzeption, um deren kritische Befragung es im Folgenden gehen soll, steht das Problem der Grenzziehung. Sie spürt der Frage nach, wie Systeme eine Grenze zu einer Umwelt hervorbringen, an der sie ihre Operationen orientieren können. Ob es sich um Maschinen, Organismen, Psychen oder soziale Systeme handelt, ist auf grundbegrifflicher Ebene zunächst nicht weiter relevant, auch wenn unterschiedliche Typen von Operation vorliegen (etwa Leben, Bewusstsein, Kommunikation), die dann spezieller Begriffe bedürfen. Ein System kann somit als etwas begriffen werden, das sich selbst eine Grenze zu einer Umwelt gibt und derart Ordnungseffekte zeitigt. (Luhmann 1984, 15ff.)

In zahlreichen Schriften hat Luhmann Prozesse der Grenzziehung und der Grenzerhaltung sozialer Systeme untersucht, wobei seine Studien zu den Funktionssystemen der modernen Gesellschaft einen zentralen Platz im Gesamtwerk einnehmen. Damit teilt die Systemtheorie ein gemeinsames Erkenntnisinteresse mit den Sozialtheorien des Poststrukturalismus, die sich ebenfalls dem Thema der Differenz widmen. Dennoch wurde von poststrukturalistischer Seite immer wieder der Vorwurf erhoben, dass die Systemtheorie fragwürdige Reinheitsvorstellungen hege und kein Gespür für die Prekarität von Differenz besitze (Koschorke 1999; Reckwitz 2004). Folgt man der Kritik, so unterstellt Luhmann dort klare Grenzverläufe, wo Autoren wie Jacques Derrida, Judith Butler, Ernesto Laclau und Gilles Deleuze auf Aufschübe und Verunreinigungen, Mehrdeutigkeiten und Vielheiten rekurrieren. Während die Systemtheorie mit ihrem Unwahrscheinlichkeitstheorem das klassische 
Problem der Möglichkeit sozialer Ordnung ins Zentrum rückt, fragen poststrukturalistische Sozialtheorien nach der Unmöglichkeit sozialer Ordnung, ohne damit partielle Ordnungseffekte leugnen zu wollen (Laclau 2002). Wenngleich diese Kritik nicht ganz von der Hand zu weisen ist, so bedient sie sich selbst einer fragwürdigen Vereindeutigung: Denn die Systemtheorie hält durchaus Begriffe und Denkfiguren bereit, um Unschärfen und Unentscheidbarkeiten zu denken. Auch systemtheoretisch lässt sich analysieren, wie soziale Ordnung von Unordnung durchzogen wird, wie sich beide zu einer spannungsreichen Einheit verschränken (siehe etwa Fuchs 1993, 198ff.; Stäheli 2008).

In diesem Zusammenhang ist erwähnenswert, dass sich Luhmann seit Mitte der 1980er Jahre immer wieder auf Michel Serres' Buch Le parasite von 1980 bezogen hat (u. a. Luhmann 1986a, 1986b). Dies mag den Beobachter zunächst überraschen, schließlich unternimmt Serres den Versuch, die Unmöglichkeit operativer Schließung und stabiler Grenzziehung aufzuzeigen. Auch die Zugangsweisen könnten kaum unterschiedlicher sein: Während Luhmann für die Soziologie eine facheinheitliche Theorie zu liefern sucht, die alles Soziale thematisieren kann, scheint Serres dem Exemplarischen und dem Figurativen stets den Vorrang vor allzu strenger Systematik und Begriffshermetik zu geben. Handelt es sich also um ein Missverständnis, eine schlichte Fehlrezeption? Oder hat man es mit jenem „Unsinn“ zu tun, den Luhmann (1997a, 46) nach eigener Auskunft in jedem seiner Bücher versteckt hat?

Beide Fragen sollen hier verneint werden. Meine These lautet: Die Figur des Parasiten erlaubt es der Systemtheorie, Grenzen und die durch sie hervorgebrachten Ordnungseffekte komplexer zu denken, als es das gängige Bild einer Demarkationslinie suggeriert. Für die Theorie maßgebliche Unterscheidungen, wie die zwischen System und Umwelt, Inklusion und Exklusion, Positivwert und Negativwert eines Codes, werden so in einer nicht-binären, mehrwertigen Weise reformulierbar. Jede Grenzziehung eröffnet demnach die Möglichkeit ihrer parasitären Subversion, die jene zugleich bestätigt und zurückweist. Ja, mehr noch: Am Beginn eines Grenzverlaufs treten notwendigerweise parasitäre Verunreinigungen auf, die auf ein Außen der Grenze verweisen. Der Parasit erhöht Ordnung und Unordnung, Negentropie und Entropie gleichermaßen. Nicht die Logik eines Nullsummenspiels, sondern ein wechselseitiges Steigerungsverhältnis beschreibt deren Zusammenhang.

Die systemtheoretische Aneignung der Figur des Parasiten gestaltet sich bei genauerer Betrachtung alles andere als unproblematisch, greift sie doch tief in die Mechanik der Theorie ein. Gegen Luhmanns häufig unsystematisch und oberflächlich bleibenden Bezugnahmen auf Serres ist zu 
betonen, dass sich der Parasit nicht bruchlos in das systemtheoretische Begriffsgefüge integrieren lässt. Insbesondere muss die operative Schließung eines Systems komplexer gedacht werden. Der vorliegende Aufsatz versucht dieses Problem zumindest im Ansatz zu skizzieren und mögliche Lösungswege aufzuzeigen. Hierbei geht es mir weniger um eine werkgeschichtliche Rekonstruktion als vielmehr um eine systematische Suchbewegung, die in dem von Luhmann entworfenen Theoriegebäude nach Anschlussstellen für die Figur des Parasiten fahndet. Die Argumentation kann sich auf die bereits vorliegende Forschungsliteratur stützen, ohne deswegen jeden Vorschlag aufgreifen zu müssen (etwa Luhmann 1995; Stäheli 1996; Nassehi 2004a; Schneider/Kusche 2010). In diesem Sinne verstehen sich die folgenden Ausführungen als ein Beitrag zu einer weiteren Öffnung der Systemtheorie für poststrukturalistische Theoriefiguren, die es in einer nicht-trivialisierenden Weise zu integrieren gilt (wichtige Studien in dieser Richtung sind Stäheli 2003; Opitz 2012).

\section{Eine Kunst der Grenzvermessung}

Luhmann begreift Systembildung als einen Prozess der Ausdifferenzierung - ein Begriff, der in mindestens zweierlei Hinsicht missverständlich ist: Erstens wird er häufig mit zunehmender Innendifferenzierung verwechselt. Zweitens suggeriert er, dass bereits eine Umwelt vorliegt, aus der sich dann ein System ,herausdifferenzieren“ kann. Beides ist aber nicht der Fall. Ausdifferenzierung bedeutet vielmehr, dass sich ein System gegen eine Umwelt schließt, also eine Grenze in die Welt einschreibt, an der es seine Operationen orientieren kann (Luhmann 1997b, 595ff.). System und Umwelt sind daher sowohl gleichursprünglich wie gleichzählig. Und wenn Luhmann gelegentlich das System als Einheit der Differenz von System und Umwelt bezeichnet, so ist dies kein Verstoß gegen syllogistische Logik, sondern ein Verweis darauf, wer hier den aktiven Part übernimmt: Das System zieht die Grenze zu seiner Umwelt, nicht umgekehrt. [1] Ist ein System nicht mehr in der Lage, die Grenze zu seiner Umwelt zu reproduzieren, so löst es sich auf. Staaten können zusammenbrechen, Ehen geschieden werden, Firmen pleitegehen, Gespräche enden. Das Auflösen einer System/Umwelt-Differenz lässt sich als Entdifferenzierung beschreiben.

Zwischen System und Umwelt herrscht ein Komplexitätsgefälle. Im operativen Außen existieren stets mehr Elemente mit entsprechend selektiver Relationierung als im Systeminneren. Die Umwelt ist daher schwächer integriert als das System, das seine Einheit wahren muss, möchte es fort-
[1] Auf die damit einhergehende Frage nach der ersten, letztlich unmöglichen Operation komme ich gleich zurück. 
bestehen (Luhmann 2009a). Gleichwohl können Systeme innerhalb ihrer Grenzen hohe Grade an Komplexität aufbauen. Ein zentraler Mechanismus ist hierbei die Systemdifferenzierung, das meint die Wiederholung von Systembildung innerhalb eines Systems. Im Unterschied zu klassischen Vorstellungen begreift Luhmann Differenzierung nicht als Dekomposition eines gegebenen Ganzen in seine Teile, sondern als das unvorhersehbare Entstehen neuer Ordnung. Die These einer Summenkonstanz wird zurückgewiesen (Luhmann 2005, 235-260). Systeme können demnach Komplexität aufbauen, indem sie in sich neue Grenzen etablieren, die zu Teilsystemen und systeminternen Umwelten führen. Mit der übergreifenden Systemeinheit weisen die Subsysteme eine gemeinsame Außengrenze auf, wenngleich sie untereinander in einem Verhältnis relativer Indifferenz stehen. Dies kann anhand der Disziplinendifferenzierung moderner Wissenschaft erläutert werden (Stichweh 1984): Da jede Wissenschaftsdisziplin Teilsystem eines Gesamtsystems ist, muss sie sich fragen lassen, inwiefern ihre Kommunikationen einerseits einen Beitrag zum wissenschaftlichen Erkenntnisgewinn leisten und sie andererseits so speziell sind, dass sie nur innerhalb eines gesonderten Faches möglich werden. Ist eine Disziplin nicht mehr in der Lage, überzeugende Antworten auf beide Fragen zu liefern, wird sie Schwierigkeiten mit ihrer doppelten Grenzziehung bekommen.

Durch den Mechanismus der Systemdifferenzierung entstehen Innendifferenzierungen. Es kommt zu einer Verschachtelung von Systemen im Sinne einer Mehrstufigkeit (siehe zu diesem Begriff Luhmann 2010, 61f.). Systeme können demnach als komplexe Ordnungsgefüge begriffen werden, die sich gegen eine überkomplexe Umwelt begrenzen müssen. Die Unordnung wird dann in die Umwelt verbannt; sie ist für das System allenfalls als Irritation erfahrbar. [2] Doch verhält es sich tatsächlich so eindeutig? Lassen sich in Luhmanns Schriften nicht auch Hinweise für eine komplexere Verhältnisbestimmung von Ordnung und Unordnung finden, die es erlaubt, operative Schließung anders zu denken und so Brücken zu Serres' Figur des Parasiten zu schlagen?

Der Begriff des Fundierungsparadoxes bietet hierzu eine interessante Möglichkeit (Luhmann 1991). Fundierungsparadoxien treten immer dann auf, wenn Systeme versuchen mit den eigenen Mitteln ihre Ausdifferenzierung zu begründen. Die Kategorien, die begründet werden sollen, werden dann zur Begründung herangezogen. Daher weisen die entsprechenden Gründungserzählungen entweder logische Fehler oder markante Lücken auf (Koschorke 2007): Die Legitimation von Rechten durch den Rekurs auf erlittenes Unrecht unterstellt, dass die Rechte schon vor ihrer Einsetzung existierten, denn sonst hätte es kein Unrecht geben können (Derrida 2013; Luhmann 2009b). Die
[2] Siehe dazu auch den Beitrag von Folkers/Lim in dieser Ausgabe. 
Gründung einer Organisation markiert ihre allererste Entscheidung, auch wenn sie diese Entscheidung gar nicht treffen konnte, da sie noch nicht gegründet war (Luhmann 2011, 123-151). Versuchen Interaktionssysteme zu klären, ob die Kommunikation unter Anwesenden bereits begonnen hat, treten ähnliche Probleme auf. Um diesen zu entgehen, existieren Begrüßungsrituale, die den Beginn einer Interaktion anzeigen sollen, hierbei aber voraussetzen müssen, dass die Interaktionsteilnehmer bereits ein gemeinsames Wahrnehmungsfeld teilen - also schon interagieren (Kieserling 1999, 110-146). Kurzum, jedes System gründet auf einem für das System selbst nicht zugänglichen Grund. [3]

In genetischer Perspektive zeigen sich Fundierungsparadoxien als unreine Anfänge (Koschorke 1999). Die erste Operation eines Systems ist stets unscharf. Sie oszilliert zwischen einem Außen, das noch nicht Umwelt geworden ist, und einem Innen, das noch nicht System geworden ist. Dennoch ereignet sie sich empirisch in der wirklichen Welt. Sie vollzieht sich als Übergang zwischen einem Nicht-mehr und einem Noch-nicht. In der retrospektiven Umschrift des Systems (siehe dazu Fuchs 1995) markiert die erste Operation die unmögliche Selbstsetzung des Systems, die faktisch dennoch möglich war, da sie von einer bereits existierenden Welt getragen wurde. Mit anderen Worten: Ein System kann seine Ausdifferenzierung gar nicht selbst angestoßen haben, auch wenn es die anfängliche Unreinheit im Nachhinein zu vergessen trachtet. Die Bedingung der Möglichkeit eines Systems wurde nicht vom System selbst generiert. Das wäre der unmögliche Übergang von null zu eins, von nichts zu etwas.

Im Umkehrschluss muss das nicht zwangsläufig zu einem ebenfalls fragwürdigen Reduktionismus führen, der keine qualitativen Übergänge denken kann. Luhmann bietet hierzu eine Alternative: eine moderate Emergenzthese. Denn es müssen stets schon Systeme ausdifferenziert sein, welche die Ausdifferenzierung neuer Systeme als eine Art „Realitätsunterbau“ (Luhmann 1984, 43, 97) tragen. Luhmann (ebd., 65ff., 157, 188, 191f., 333) hat dieses Problem als mutualistische beziehungsweise multiple Konstitution auf den Begriff gebracht. Für soziale Systeme bedeutet dies: Damit doppelte Kontingenz entstehen kann, bedarf es mindestens zweier Sinnsysteme - Ego und Alter -, welche ihre Verhaltensselektionen wechselseitig voneinander abhängig machen und dabei unterstellen, dass weder die eigenen noch die fremden Selektionen vollständig determiniert sind. Ego und Alter sind daher die mundane Möglichkeitsbedingung für die Ausdifferenzierung eines neuen Sozialsystems, das als „emergente Ebene des Zwischensystemkontakts“ (ebd., 159) zu begreifen ist.
[3] Dieses Problem existiert bereits auf der Ebene jeder einzelnen Form, Beobachtung, Operation: Versucht eine Form mit der eigenen Unterscheidung die Einheit der Unterscheidung zu bestimmen, möchte eine Beobachtung sich selbst hinsichtlich ihres blinden Flecks beobachten, und strebt eine Operation danach, sich selbst zu bewirken, so entsteht eine Paradoxie. Das ist nur eine andere Weise zu sagen, dass keine Form, Beobachtung, Operation sich vollständig schließen kann. Stets gibt es eine teilweise Öffnung zu einem Außen, die die Schließung unterbricht. 
Luhmanns differenzierungstheoretisches Vorgehen gleicht somit dem einer Grenzvermessung: Schritt für Schritt werden Systemgrenzen abgegangen, um sie in ihrer sozialen, sachlichen, zeitlichen (und leider viel zu selten auch räumlichen) Dimension zu beschreiben. An seinem Beginn verwirrt sich jedoch der Grenzverlauf. Die Theorie stößt dann auf eine merkwürdige Kreuzung der behutsam abgeschrittenen Systemgrenze mit anderen Systemgrenzen. Die Verwirrung der Linienführung schafft eine uneindeutige Situation, in der Raum für Neues entsteht. Die erste Operation eines Systems kann daher als das ausgeschlossene, eingeschlossene Dritte derjenigen Systeme verstanden werden, die die Ausdifferenzierung ermöglicht haben. Neue Ordnungen zeichnen sich stets an der Grenze bereits bestehender Ordnungen ab. Jedes System entsteht in einer Nische.

\section{Was sich der Grenzziehung nicht fügt}

Genau an dieser Stelle setzt Serres mit der Figur des Parasiten ein. Sein methodisches Vorgehen begreift er denn auch nicht als Grenzvermessung, sondern als randonnée, was so viel wie ein Umherwandern, eine „interessante Reise“ (Serres 1987, 15) bedeutet. Demnach soll all den Momenten nachgegangen werden, die sich einer Grenzziehung nicht fügen, auch wenn sie auf diese verwiesen bleiben. Serres versucht zu zeigen,

„[d]aß es überall Unterbrecher gibt, die mit großem Aufwand daran arbeiten abzuzweigen und zu unterschlagen, was da über die Wege wandert. Die Bezeichnung, die man diesen verbreiteten und vielfältigen Aktivitäten zumeist beilegt, lautet Parasitentum, und ich fürchte, es ist die gewöhnlichste Sache von der Welt.“ (Ebd., 24)

Jede Ordnung weist somit Löcher und Risse auf, die es sichtbar zu machen gilt. Eine vollständige Schließung, ein perfektes System kann es nicht geben. Aber umgekehrt ist ebenso richtig, dass das Loch einen Rand und der Riss einen Körper voraussetzt. Ordnung und Unordnung verschränken sich notwendigerweise ineinander - so die These. Doch was hat es mit dem Wort ,Parasit‘ auf sich, das zumeist äußerst negative Konnotationen trägt? Wie möchte es Serres in Abgrenzung zu diesen verstanden wissen? Und inwiefern kann die Systemtheorie die Serres'sche Argumentation für sich fruchtbar machen? 
Der griechischen Herkunftsbedeutung folgend, meint der Parasit einen Mitesser. Das Wort bezeichnet jemanden, der sich aus einer Nebenposition heraus (pará) ernährt (sitos). In der klassischen Antike wurden zunächst die Inhaber eines religiösen Amtes mit diesem Ausdruck belegt; das war insbesondere bei den in Attika beheimateten Kulten der Fall. Als Vertreter ihrer Polis hatten die Parasiten das Opfermahl mit den Göttern zu verspeisen. Seit der Mitte des 4. Jahrhundert v. u. Z. erhielt das Wort Einzug in die athenische Theaterwelt, wodurch es eine Umwertung erfuhr. Als ,Parasit' wurde nunmehr eine Bühnenfigur bezeichnet, die sich als ungeladener Gast einen Platz an der Tafel anderer Leute zu verschaffen suchte. (Nesselrath 2000; Pernerstorfer 2012; Enzensberger 2001, 11-72) Zu einem biologischen Begriff wurde das Wort erst in der Mitte des 17. Jahrhunderts, bevor es verstärkt im 19. Jahrhundert in medizinischen Diskursen zu zirkulieren begann. Parallel kam es zu einem abermaligen Bezug auf die Sozialwelt. Unter anderem verbreitete sich die Rede vom ,jüdischen Parasiten ‘ - ein Motiv, das im Nationalsozialismus den Ausschluss, die Vertreibung und den Mord von Millionen Juden zu legitimieren half. Und auch heutzutage finden sich in öffentlichen Debatten stark abwertende Klagen über ,Schmarotzer' und ,Sozialparasiten', welche der Allgemeinheit schadeten. (Enzensberger 2001, 92-275; Musolff 2011)

Michel Serres' Buch Der Parasit markiert eine abermalige Wende in der Geschichte dieses Wortes, zeigt die Argumentation doch auf, dass parasitäre Verunreinigungen ein allgemeines und letztlich unvermeidbares Phänomen sind. Auf jedem Ordnungsniveau existieren Parasiten, die von Serres als die Rückkehr des Ausgeschlossenen ins Innere beschrieben werden. Überall dort, wo ein ordnungsbildender Ausschluss stattfindet, können sie vorkommen. Diese These ist durchaus als eine Kritik zu lesen: Wenn die parasitäre Störung den Normalfall bildet, verlieren unterkomplexe Reinheitsvorstellungen ihre Überzeugungskraft. Die Figur des Parasiten erweist sich so als ein Sinnbild für die Verschränkung von Ordnung und Unordnung (Serres 1987, 131). Ob es sich hierbei um Maschinen, Organismen, Psychen, Sozialstrukturen oder logische Formen handelt, ist zunächst nicht weiter relevant. Serres' Parasit setzt ähnlich allgemein an wie Luhmanns System. Bei genauerer Betrachtung zeigt sich, dass die beiden Zugänge logisch kompatibel sind und in gewisser Hinsicht einander implizieren (Stäheli 1996, 66-78). „Kein System ohne Parasit“, heißt es apodiktisch bei Serres $(1987,26)$, der seine Überlegungen ebenfalls in einem informations-, evolutions- und systemtheoretischen Rahmen entwickelt. „Aber auch, kein Parasit ohne System“, ließe sich im Geiste Luhmanns ergänzen. 
Wie Petra Gehring (2010) betont, präsentiert Serres die Figur des Parasiten mithilfe einer Vielzahl von Figuren. Damit soll gesagt sein, dass er anhand einer Fülle von literarischen Versatzstücken, philosophischen Theoremen, kulturellen Formen, naturwissenschaftlichen Phänomenen und anderem mehr die Elemente eines allgemeinen Ordnungsproblems herauspräpariert - eines Problems, das all diese besonderen Bereiche übergreift und in ein Kontinuum stellt. Der Parasit ist überall, aber immer anders. Ebenso vielfältig sind die Figuren, die Serres anführt, um letztlich ein gemeinsames Grundmotiv aufscheinen zu lassen. Die Systemtheorie Luhmanns kann jedoch bei einer solch figurativen Darstellung nicht verbleiben. Sie ist durch und durch auf Theorie geeicht, sofern man darunter eine Menge aufeinander abgestimmter Begriffe versteht. Daher sollen im Folgenden zunächst vier Elemente der Serres'schen Figur des Parasiten herausdestilliert werden, die so etwas wie das Grundgerüst bilden, bevor im Anschluss zu fragen ist, wie sich diese in einem systemtheoretischen Begriff reformulieren lassen.

Der Parasit ist erstens durch Paradoxalität gekennzeichnet. Mit einer Formulierung Serres' (1987, 41ff.) kann er als der „ausgeschlossene, eingeschlossene Dritte“ beschrieben werden. Das ist keine Wortspielerei, sondern der Versuch einer schwierigen Verhältnisbestimmung. Der Parasit meint eine eigentümliche Relation zu einer Relation, genauer: Als nicht vorgesehener Dritter nährt er sich von einem bestehenden Produktions- oder Austauschverhältnis, das er zugleich bestätigt wie zurückweist. Die parasitäre Relation akzeptiert und unterläuft die Wirtsrelation (ebd., 29). Auf diese Weise kommt Rauschen in das System, dessen Grenzen werden brüchig und uneindeutig. [4] Die Stadtratte hat ihren Vetter die Landrate zum Mahl eingeladen - so das Eingangsbeispiel Serres' -, verspeist werden die Lebensmittel des Steuerpächters, der sich selbst seinen Teil vom Produzenten abgezweigt hat. Der Parasit ist der ungebetene Gast, der mitisst. Er ist der Ausgeschlossene, der ins Innere zurückkehrt (ebd., 118f., 226). Daraus resultiert seine Unruhe. Schon beim ersten Knarren der Tür rennen die Ratten auseinander, in der Angst entdeckt zu werden.

Zweitens ist die parasitäre Beziehung gerichtet. Der eine nimmt, ohne zu geben. Der andere gibt, ohne einen Gegenwert zu erhalten. „Parasitär nenne ich eben dieses Halbleiterphänomen, dies Ventil, diesen einfachen Pfeil, diese Beziehung, die nur eine Richtung kennt.“ (Ebd., 14) Der Parasit unterbricht jede Reziprozität. Ohne Gegenleistung eignet er sich ein Gut an, das andere hergestellt haben. Produktions- und Tauschlogik liegen dem Parasiten fern, auch wenn er funktionierende Produktions- und Tauschverhältnisse voraussetzt. In gewisser Weise verkörpert er das Gegenmodell
[4] Benötigt wird daher eine nicht-klassische Logik, die mit Paradoxien und Unschärfen umgehen kann (Serres 1987, 88f.). 
zur Gabe: das reine Nehmen. Und falls der Parasit doch einmal tauschen sollte, so wechselt er die Münzart. Das Speisen an der Tafel anderer Leute wird mit Scherzen und Geschichten beglichen. Der Parasit „diagonalisiert“ den Tausch (ebd., 57-65).

Die Figur des Parasiten beschreibt drittens einen reflexiven Mechanismus. [5] Parasitäre Ordnungen sind parasitierbare Ordnungen. Des einen Laus ist des anderen Wirt. Parasiten unterwandern nicht nur Grenzen, sie verfügen auch über eigene; dies macht sie zum möglichen Gegenstand von Grenzverletzungen. ,Parasitär ${ }^{\star}$ bezeichnet somit keine ontologische Eigenschaft, sondern eine relationale Position. Der Steuerpächter nährt sich vom Produzenten, die Stadtratte findet ihre Speisen in der Kammer des Steuerpächters, die Landratte schmarotzt bei ihrem Vetter in der Stadt. Zusammen bilden sie eine parasitäre Kaskade, eine Kette von „aufeinandergepfropften Parasiten“ (ebd., 25), in der jeder versucht der letzte zu sein. Die eigenen Grenzen sollen intakt bleiben, auch wenn an denen der anderen fleißig genagt wird. Gleichzeitig ist es attraktiv, möglichst nah an der Produktionsquelle zu sitzen, obgleich dadurch die Gefahr steigt, selbst zum Ziel parasitärer Unterwanderungen zu werden. Das Ergebnis ist eine nicht still zu stellende Dynamik in der parasitären Kaskade. Fortwährend vollziehen sich Positionswechsel (ebd., 82).

Viertens lässt sich nicht immer eindeutig und verbindlich entscheiden, wer lhôte im Sinne von Wirt und wer l'hôte im Sinne von Gast ist (ebd., 31ff.). Das Französische ist in diesem Punkt genauso unentschieden wie die Sache, die es zur Sprache bringt. Die Verortung des parasitären Rauschens ist somit abhängig von der „Stellung des Beobachters“ (ebd., 102), der Parasit ist an eine Perspektive gebunden. Serres führt hierzu das Beispiel einer Abendgesellschaft an, bei der die Unterhaltung der Gäste durch das Klingeln des Telefons kurzfristig unterbrochen wird: „Sobald ich das Gespräch aufnehme, setzt die Unterhaltung an der Festtafel wieder ein, sie wird zum Lärm, zum Rauschen, für das neue Wir. Das System schaukelt. Nähere ich mich wieder der Tafel, so wird der Lärm nach und nach wieder zur Konversation.“ (Ebd.) Was Lärm und was Information ist, wird von der Position des Hörers im Raum mitbestimmt. Wer produziert und wer konsumiert, wer nimmt und wem genommen wird, in welche Richtung die parasitäre Kaskade somit verläuft, ist nur durch die Angabe einer kontingenten Beobachterposition zu klären. Unterschiedliche Zuordnungen können die Folge sein.

Mit seinen vier Grundmerkmalen - Paradoxalität, Gerichtetheit, Reflexivität, Perspektivität verweist der Parasit auf Relationen und Grenzen, die er sowohl bestätigt als auch zurückweist. Ordnung und Unordnung werden von ihm gleichermaßen gestiftet. Systemtheoretisch lässt sich dieser
[5] Das meint einen Mechanismus, der auf sich selbst anwendbar ist (Luhmann 2009c). 
Gedanke wie folgt reformulieren: [6] Parasitäre Relationen können überall dort entstehen, wo sich Elemente zu einem System verknüpfen. [7] Jede Ausdifferenzierung einer System/Umwelt-Differenz ermöglicht das Einnisten spezifischer Parasiten, welche die Grenzziehung konsolidieren und unterlaufen. Es handelt sich dann weder um eine reibungslose Differenzierung noch um eine vollständige Entdifferenzierung. Der Parasit sperrt sich gegen solch eindeutige Zuordnung. Er operiert paradox, da er sich eine etablierte System/Umwelt-Grenze zunutze macht, sie aber zugleich verwirrt. Die Grenze verschwimmt, sie wird uneindeutig. Die volle operative Schließung des Systems wird dadurch verhindert, auch wenn eine ausreichend starke Schließungsbewegung vorausgesetzt bleibt. Der Parasit ist das Dritte zwischen Differenzierung und Entdifferenzierung.

Diese formale Begriffsbestimmung eignet sich für Spezifizierungen in mehrere Richtungen. Zunächst ist sie hinsichtlich verschiedener Systemtypen zu konkretisieren. Technische, biologische, psychische und soziale Parasiten besitzen einen jeweils anderen Charakter, da es sich um unterschiedliche Formen von Operation und Grenzziehung handelt, auf die sie bezogen sind. In der soziologischen Diskussion wurden bislang vor allem codierte Sozialsysteme betrachtet (etwa Stäheli 2003, 303ff.; Schneider 2008, 181f.; Opitz 2012, 251ff.). Aber auch nicht-codierte Systeme sind parasitierbar, weshalb die obige Begriffsbestimmung ihren Ausgang vom allgemeineren Problem der Grenzziehung genommen hat. Codierung ist nur eine Möglichkeit, wie ein System sich operativ schließen kann. Ferner bedarf weiterer Klärung, inwiefern Parasiten notwendigerweise auftreten müssen, was von Serres immer wieder suggeriert wird. Zwar leuchtet es intuitiv ein, dass die Wahrscheinlichkeit von parasitären Grenzverunreinigungen mit der Zeit zunimmt, worauf auch der Entropiegedanke verweist (Serres 1987, 285ff.). Ebenfalls besitzt die erste Operation eines Systems, wie bereits gezeigt wurde, stets einen parasitären Charakter. Dennoch ist das Auftreten einer solchen Operation nicht notwendig, sondern mit einem Wahrscheinlichkeitswert versehen, der kleiner als eins ist. Nur weil der Steuerpächter Lebensmittel hortet, müssen diese noch lange nicht von Ratten verspeist werden. Türen, Schränke und Fallen versuchen das zu unterbinden. Es wäre dann nach den Bedingungen zu fragen, die das Entstehen einer parasitären Verunreinigung wahrscheinlich oder unwahrscheinlich werden lassen. Ebenso ist zu unterscheiden, ob Parasiten lediglich situativ anfallen oder sich verstetigen und zu eigener Systembildung führen (Schneider 2008, 182). Hierbei kann vermutet werden, dass ein enger Zusammenhang zwischen dem Grad an Ausdifferenzierung
[6] Die folgende Begriffsbestimmung orientiert sich an der System/Umwelt-Differenz. Der Formbegriff im Anschluss an George Spencer-Brown ließe eine noch allgemeinere Bestimmung des Parasiten zu, da Systemdifferenzierung ein Spezialfall von Formdifferenzierung ist (Karafillidis 2010, 243-347). Demnach sind nicht nur ganze Systeme, sondern auch sachliche, soziale, zeitliche und räumliche Differenzierungen unterhalb der Systemschwelle parasitierbar.

[7] Die Letztelemente eines Systems können durch einen Beobachter selbst wiederum als eine Relationierung von Elementen auf einem niedrigeren Emergenzniveau dekomponiert werden. Systeme erweisen sich so als eine Relationierung von Relationen (Luhmann 1984, 41ff.). 
einer parasitären Relation und der Wahrscheinlichkeit ihrer neuerlichen parasitären Unterwanderung besteht.

Das sind einige der Grundprobleme einer - wenn man so möchte - systemtheoretischen Parasitologie. Auch wenn der vorliegende Aufsatz eine solche nicht voll entwickeln kann, soll doch im Folgenden ihr gesellschaftstheoretisches Potenzial aufgezeigt werden. Da es sich bei der von Luhmann entwickelten Systemtheorie, trotz aller Ausgriffe in andere Disziplinen, im Kern um einen Beitrag zum soziologischen Erkenntnisgewinn handelt, hat sich der Begriff des Parasiten nicht zuletzt für das Gesellschaftssystem als instruktiv zu erweisen. Denn mit diesem ist das umfassende Sozialsystem adressiert, das die Freiheitsspielräume aller anderen Sozialsysteme konditioniert. Der Begriff des Parasiten, so die hier vertretene Auffassung, kann dabei helfen, Luhmanns Gesellschaftstheorie in ihrer Bauweise reichhaltiger und in ihrem Gegenstandsbezug angemessener zu gestalten. Nicht nur die Ordnung, auch die Unordnung der Gesellschaft gerät dann in den Blick.

\section{Die (Un-)Ordnung der Gesellschaft}

Unter Gesellschaft versteht Luhmann (2009d) das umfassende Sozialsystem, das alle anderen sozialen Systeme einschließt. Daher besitzt die Systemgrenze der Gesellschaft, die an der Unterscheidung zwischen Kommunikation und Nicht-Kommunikation orientiert ist, für Interaktionen, Organisationen und Konflikte einen bindenden Charakter. Nur innerhalb der Gesellschaft können Gespräche stattfinden, Firmen ihre Kunden werben und Streitigkeiten ausgetragen werden (Luhmann 2009e). Dennoch sind diese Sozialsysteme nicht deckungsgleich mit dem Gesellschaftssystem, da ihre Grenzen deutlich enger gesteckt sind. Sie vollziehen Gesellschaft mit und bleiben dennoch von ihr unterscheidbar.

Die moderne Gesellschaft sieht Luhmann durch einen Primat funktionaler Differenzierung gekennzeichnet (siehe neben Luhmanns umfassenden Monographien zu den meisten der Funktionssysteme auch Mayntz et al. 1988; Nassehi 2004b). Demnach gewinnt sie durch das Nebeneinander sachlich spezialisierter Subsysteme (wie Recht, Massenmedien, Politik, Kunst, Ökonomie, Wissenschaft, Erziehung) an Struktur. Die funktional differenzierten Teilsysteme sind füreinander nicht substituierbar und können in keine gesellschaftsweite Rangfolge gebracht werden - aber genau aus diesem Grund bleiben sie aufeinander verwiesen. Die Einheit der Gesellschaft besteht strukturell 
nur noch in der Differenz der Funktionssysteme. Es ist dieses Trennungsmuster, das den gesellschaftlichen Zusammenhang stiftet. Luhmanns gesamte Gesellschaftstheorie ist letztlich dem Versuch gewidmet, die These einer funktionalen Primärdifferenzierung der modernen Gesellschaft zu plausibilisieren. Ein Großteil der hierzu vorgetragenen Argumente vermag zu überzeugen. Dennoch existieren Probleme und offene Fragen, von denen insbesondere drei für den hier interessierenden Zusammenhang wichtig sind.

Erstens vertritt die Systemtheorie die Auffassung, dass die moderne Gesellschaft als Weltgesellschaft ausdifferenziert ist (Luhmann 2009f; Stichweh 2000; Greve/Heintz 2005). Demnach gibt es weltweit nur noch ein einziges Gesellschaftssystem. Der Horizont füreinander erreichbarer Kommunikationen hat sich zu einem globalen ausgeweitet. Dieser Prozess beginnt mit der Frühen Neuzeit, unter anderem eingeleitet durch Kolonialismus und globale Arbeitsteilung, und setzt sich in der Moderne so weit durch, dass er vorerst als irreversibel erscheint. Die Rede von einer deutschen, pakistanischen oder brasilianischen Gesellschaft verliert damit ihre Berechtigung. In einigen späten Arbeiten hat Luhmann (2009g; 1999; 1995) jedoch selbstkritisch Bedenken geäußert, ob die Weltgesellschaft tatsächlich mittels des Prinzips funktionaler Differenzierung adäquat beschrieben werden kann. Einerseits kündige sich bereits eine Primärdifferenzierung der Weltgesellschaft nach Maßgabe der Unterscheidung zwischen Inklusion und Exklusion an; andererseits verhinderten parasitäre Netzwerke in der Semi-Peripherie der Weltgesellschaft die volle Realisierung funktionaler Differenzierung. Die von den Funktionssystemen und ihren Organisationen hervorgebrachten Produkte (wie politische Macht, ökonomischer Reichtum, mediale Aufmerksamkeit, rechtliche Entscheidungsgewalt) würden von klientelistischen, formal ausgeschlossenen Netzwerken angeeignet. Dies hat in der Systemtheorie eine Debatte angestoßen, die als gegenwärtig noch weitestgehend offen betrachtet werden kann (siehe etwa Holzer 2007; Japp 2007; Leanza 2014 [i. E.]). Was auf dem Spiel steht, ist nichts weniger als die These von der funktionalen Primärdifferenzierung der Weltgesellschaft. Wahrscheinlich muss sie zugunsten von Beschreibungen aufgegeben werden, die historisch und regional deutlich feingliedriger ansetzen. Hierzu könnte sich der Begriff des Parasiten als fruchtbar erweisen, da er die ,Gleichzeitigkeit des Ungleichzeitigen' thematisierbar macht.

Zweitens vernachlässigt Luhmanns Gesellschaftstheorie Phänomene und Mechanismen sozialer Ungleichheit in der Moderne. Stratifikation erscheint auf der Ebene des Gesellschaftssystems entweder als ein vormodernes Strukturmuster oder als eine nicht weiter zu beachtende Größe (sie- 
he für diese Kritik Schwinn 2004; Schroer 2010). Die These einer funktionalen Primärdifferenzierung der Weltgesellschaft schließe zwar die Existenz genuin moderner Stratifikationsformen nicht aus, seien für ganze Gesellschaftssysteme doch „Gemengelagen mehrerer Differenzierungsformen typisch“ (Luhmann 1997b, 612). Dennoch ließe sich zeigen, dass diese lediglich als „Nebenprodukte der Eigendynamik der Funktionssysteme“ (ebd.) abfielen. Selbst wenn man sich hierbei auf die Kernregionen funktionaler Differenzierung beschränkt, ist jedoch mehr als fraglich, ob eine solche Herangehensweise der Bedeutung sozialer Ungleichheit für die moderne Gesellschaft tatsächlich gerecht wird. Ist nicht vielmehr davon auszugehen, dass die Kategorien sozialer Ungleichheit (wie Klasse, Geschlecht, Ethnizität) unter den Bedingungen funktionaler Differenzierung zwar formal weitestgehend marginalisiert sind, aber gleichzeitig eine faktische Prägekraft für individuelle Karrieren und kollektive Lagen aufweisen? [8] Sollte die Antwort positiv ausfallen, könnte der Begriff des Parasiten ein wichtiges Beschreibungsinstrumentarium liefern: Die Kategorien sozialer Ungleichheit stehen demnach in einem parasitären Verhältnis zu den Funktionssystemen der modernen Gesellschaft und ihren Organisationen. Die ungleich verteilten Ressourcen werden zwar von den funktionalen Teilsystemen hergestellt, aber bisweilen nach Maßgabe von Kriterien verteilt, die durch diese Differenzierungsform ausgeschlossen sind (siehe für erste Analysen in diese Richtung Ohlendieck 2003; Nassehi 2004a; Schneider/Kusche 2010). Die Kategorien sozialer Ungleichheit sind daher weder vormoderne Relikte noch fügen sie sich bruchlos in die strukturelle Grundsignatur der Moderne. Eher sollten sie als „die dunkle Kehrseite der bewußten, klaren Organisation“ begriffen werden, als „die dunklen Flecken des Systems“ (Serres 1987, 25). Dies kann auch über den Protest aufklären, der ihnen regelmäßig entgegengebracht wird.

Drittens scheint es in der von Luhmann beschriebenen Gesellschaft keine Kriege zu geben. Zwar wird in historischen Einschüben gelegentlich auf kriegerische Konflikte verwiesen. Dennoch ist dieses für die Gesellschaftsgeschichte so wichtige Thema im Theoriegebäude nicht verankert, weshalb es unbegriffen bleibt. Wie Hans Joas und Wolfgang Knöbl (2008) herausgearbeitet haben, handelt es sich hierbei um ein allgemeines Problem der Soziologie, das keineswegs auf die Systemtheorie beschränkt ist. Die relative Abwesenheit von Kriegen in Luhmanns Schriften hat also nur sehr bedingt mit der vermeintlichen Konfliktferne systemtheoretischen Denkens zu tun, im Gegenteil: In den Sozialen Systemen wird in einem umfangreichen Kapitel das Konzept eines parasitären Konfliktsystems entwickelt, das in der Gesellschaftstheorie jedoch kaum Verwendung findet. Ein Kon-
[8] Formale Marginalisierung soll heißen: Die Differenzierung nach Klassen, Geschlechtern und Ethnien ist in der modernen Gesellschaft solange legitim, wie sie auf der Ebene der Interaktion verbleibt. Nur dürfen diese Kategorien nicht - mit wenigen Ausnahmen - für die Operationen von Funktionssystemen und Organisationen leitend sein, da mit ihnen Prozesse der Statuszuweisung verbunden sind. Dass es sich faktisch aber anders verhält, wie die empirische Ungleichheitsforschung immer wieder nachweist, macht das Skandalon dieser Kategorien aus. Siehe dazu jetzt auch Itschert (2013). 
fliktsystem gelangt immer dann zur Ausdifferenzierung, wenn in der Kommunikation eine Zurückweisung zurückgewiesen, eine Negation negiert wird. So entsteht Gegnerschaft, die systembildend wirkt. (Luhmann 1984, 488-550; Kieserling 1999, 257-302) Unabhängig vom Anlass, der trivial gewesen sein mag, kann sich ein Konflikt auf diese Weise reproduzieren und zahlreiche neue Themen integrieren. Überhaupt tendieren Konflikte dazu, den anfänglichen Widerspruch in der Sache in die Sozialdimension zu verschieben, was sowohl ihre thematische Flexibilität wie ihre prinzipielle Gewaltnähe erklärt. Mit der Zeit wird der Andere, der sich dem eigenen Willen nicht fügt, zum eigentlichen Ärgernis.

Stets finden Konflikte ihre Auslöser in bereits ausdifferenzierten Sozialsystemen. Diese werden von jenen als parasitäre Nische genutzt und ausgehöhlt (Luhmann 1984, 532f.). Mitunter überlebt der Konflikt sein Wirtssystem, nachdem er es vollständig zerstört hat. Der Ehestreit lässt sich auch nach der Scheidung weiterführen. Er produziert seine eigene Wirklichkeit, die für Außenstehende oft nicht nachvollziehbar ist. Wolfgang Ludwig Schneider (2008, 185-189; siehe für ein systemtheoretisches Verständnis von Militär und Krieg auch Kohl 2009; Kuchler 2013) hat nun den Vorschlag gemacht, moderne Kriege als parasitäre Konfliktsysteme des politischen Systems zu verstehen. Demnach überführen sie in Abhängigkeit von ihrer konkreten Form (Staatenkrieg, Guerillakrieg, Bürgerkrieg etc.) auf je spezifische Weise die politisch ohnehin ablaufenden Konflikte in eine gewalttätige Auseinandersetzung, die dann in der Regel weitere Konfliktlinien generiert. Ein Krieg gelangt zu seiner vollen Ausdifferenzierung, wenn das rechtlich und moralisch codierte Tötungsverbot fällt. Wer als Kämpfer an Kriegshandlungen teilnimmt, muss damit rechnen, getötet zu werden. Das Töten orientiert sich dabei an der Differenz von Freund und Feind, in vielen Fällen auch an der von Kombattant und Zivilist. Als äußerst aggressiver Parasit zerstört das kriegerische Konfliktsystem die organischen, psychischen und sozialen Systeme in seiner Umwelt. Es hinterlässt Trümmer der verschiedensten Art.

Alle drei Problemfelder besitzen einen gemeinsamen Fluchtpunkt: An die Stelle einer allzu glatten Gesellschaftsbeschreibung, die jedem System einen festen Platz im Differenzierungsgefüge zuweist, treten parasitäre Verstrickungen und Grenzverwischungen. Das Nebeneinander der Funktionssysteme wird durch das Ineinander, ja Durcheinander verschiedener Systemtypen und Differenzierungsformen herausgefordert. Gesellschaft meint die umfassende Sozial(un)ordnung. Ihre Struktur ist unscharf. 


\section{Zwischen Hermes und Pfingsten}

Wie sichtbar wurde, gestaltet sich die systemtheoretische Aneignung der Figur des Parasiten alles andere als unproblematisch, greift sie doch tief in die Mechanik der Theorie ein. Neben Innenseite und Außenseite einer Unterscheidung ist nunmehr ein weiteres Glied in die differenztheoretische Kette einzufügen: das ausgeschlossene, eingeschlossene Dritte. Die bei Luhmann häufig binär angelegten Leitunterscheidungen müssen dann anders gedacht werden. In das Konzept operativer Schließung sind Unterbrecher einzuführen, die die Grenzziehung uneindeutig werden lassen. Daher gilt es kritisch zu fragen, inwiefern die Figur des Parasiten in der Systemtheorie selbst ein parasitäres Dasein zu fristen hat, solange eine Umschrift hin zu einem mehrwertigen Grenzbegriff noch nicht vollzogen worden ist.

Einer solchen Umschrift mag in der Systemtheorie ein Denkmotiv entgegenstehen, das mit Serres $(1987,73)$ als „Pfingstschema“ bezeichnet werden kann. Während der griechische Götterbote Hermes als Überbringer von Nachrichten fungiert, beschreibt das christliche Pfingstereignis eine Kommunikation ohne Mittler und Verteiler (Röttgers 1999). Es meint eine Situation, in der „jedes beliebige Element mit jedem anderen in Beziehung treten könnte, ohne auf einen Vermittler angewiesen zu sein“ (Serres 1987, 73). Systemtheoretisch entspräche dies einer vollständigen Entdifferenzierung bei gleichzeitiger Totalintegration. Es ist kein Zufall, dass eine solche Vorstellung im religiösen Kosmos beheimatet ist; ein an Differenzierung interessierter Beobachter kann mit ihr nur wenig anfangen. Insofern folgt die Systemtheorie entschieden dem Modell des Hermes. An die Stelle von Einheit tritt Differenz. Pfingsten bildet in systemtheoretischer Perspektive daher nichts anderes als ein Thema religiöser Kommunikation, operativ ist diese durch Immanenz, Selektivität und Grenzbildung gekennzeichnet (Luhmann 2002). Hermes muss die frohe Botschaft überbringen, sie verbreitet sich nicht selbst. Trotz dieses - man ist versucht zu sagen - Bekenntnisses zum Medialen und Mediatisierten erwecken systemtheoretische Texte nicht selten den Eindruck, dass die Prozesse der Grenzziehung eindeutig, fehlerfrei und unumstritten vonstattengehen. Die Systemgrenze wird scheinbar nicht durch Mittler unterbrochen; hier lässt sich jeden Tag Pfingsten feiern. Die operative Schließung des Systems ist perfekt. Die Linie hat sich selbst zu fassen bekommen, sie ist Kreis geworden. Möchte man diesem Denkmotiv entsagen, muss Differenz stärker problematisiert werden. Der Begriff des Parasiten leistet genau dies. 


\section{Bibliographie}

Derrida, J. (2013) Gesetzeskraft. Der „mystische Grund der Autorität“. Frankfurt a. M.: Suhrkamp.

Enzensberger, U. (2001) Parasiten. Ein Sachbuch. Frankfurt a. M.: Eichborn.

Fuchs, P. (1993) Moderne Kommunikation. Zur Theorie des operativen Displacements. Frankfurt a. M.: Suhrkamp.

Fuchs, P. (1995) Die Umschrift. Zwei kommunikationstheoretische Studien: „japanische Kommunikation“ und

„Autismus“. Frankfurt a. M.: Suhrkamp.

Gehring, P. (2010) Der Parasit. Figurenfülle und strenge Permutation. In: Eßlinger, E.; Schlechtriemen, T.;

Schweitzer, D.; Zons, A. (eds.) Die Figur des Dritten. Ein kulturwissenschaftliches Paradigma. Frankfurt a. M.:

Suhrkamp.

Greve, J.; Heintz, B. (2005) Die „Entdeckung“ der Weltgesellschaft. Entstehung und Grenzen der

Weltgesellschaftstheorie. In: Zeitschrift für Soziologie. Sonderheft „Weltgesellschaft“: 89-119.

Holzer, B. (2007) Wie „modern“ ist die Weltgesellschaft? Funktionale Differenzierung und ihre Alternativen. In: Soziale Systeme 13(1+2): 357-368.

Itschert, A. (2013) Jenseits des Leistungsprinzips. Soziale Ungleichheit in der funktional differenzierten Gesellschaft. Bielefeld: transcript.

Japp, K. P. (2007) Regionen und Differenzierung. In: Soziale Systeme 13(1+2): 185-195.

Joas, H.; Knöbl, W. (2008) Kriegsverdrängung. Ein Problem in der Geschichte der Sozialtheorie. Frankfurt a. M.: Suhrkamp.

Karafillidis, A. (2010) Soziale Formen. Fortführung eines soziologischen Programms. Bielefeld: transcript.

Kieserling, A. (1999) Kommunikation unter Anwesenden. Studien über Interaktionssysteme. Frankfurt a. M.: Suhrkamp.

Kohl, T. (2009) Zum Militär der Politik. In: Soziale Systeme 15(1): 160-188.

Koschorke, A. (1999) Die Grenzen des Systems und die Rhetorik der Systemtheorie. In: Ders.; Vismann, C. (eds.) Widerstände der Systemtheorie. Kulturtheoretische Überlegungen zum Werk Niklas Luhmanns. Berlin: Akademie.

Koschorke, A. (2007) Zur Logik kultureller Gründungserzählungen. In: Zeitschrift für Ideengeschichte 2(1): 5-12.

Kuchler, B. (2013) Kriege. Eine Gesellschaftstheorie gewaltsamer Konflikte. Frankfurt a. M.; New York: Campus.

Laclau, E. (2002) Emanzipation und Differenz. Wien: Turia + Kant.

Leanza, M. (2014 [i. E.]) Zentren und Ränder funktionaler Differenzierung. Niklas Luhmanns Theorie der modernen Gesellschaft. In: Farzin, S.; Laux, H. (eds.) Gründungsszenen soziologischer Theorie, Wiesbaden: VS.

Luhmann, N. (1984) Soziale Systeme. Grundriß einer allgemeinen Theorie. Frankfurt a. M.: Suhrkamp.

Luhmann, N. (1986a) Ökologische Kommunikation. Kann die moderne Gesellschaft sich auf ökologische Gefährdungen einstellen? Opladen: Westdeutscher Verlag. 
Luhmann, N. (1986b) „Distinctions directrices“. Über die Codierung von Semantiken und Systemen. In: Neidhardt, F.; Lepsius, M. R.; Weiß, J. (eds.) Kultur und Gesellschaft. Sonderheft 27 der Kölner Zeitschrift für Soziologie und Sozialpsychologie: 145-161.

Luhmann, N. (1991) Sthenographie und Euryalistik. In: Gumbrecht, H. U.; Pfeiffer, K. L. (eds.) Paradoxien,

Dissonanzen, Zusammenbrüche. Situationen offener Epistemologie. Frankfurt a. M.: Suhrkamp.

Luhmann, N. (1993) Wie ist soziale Ordnung möglich? In: Ders. Gesellschaftsstruktur und Semantik. Studien zur Wissenssoziologie der modernen Gesellschaft. Band 2. Frankfurt a. M.: Suhrkamp.

Luhmann, N. (1995) Kausalität im Süden. In: Soziale Systeme 1(1): 9-28.

Luhmann, N. (1997a) Biographie im Interview. Gespräch am 8. Januar 1996 in Oerlinghausen. In: Horster, D. Niklas Luhmann. München: Beck.

Luhmann, N. (1997b) Die Gesellschaft der Gesellschaft. Frankfurt a. M.: Suhrkamp.

Luhmann, N. (1999) Jenseits von Barbarei. In: Ders. Gesellschaftsstruktur und Semantik. Studien zur Wissenssoziologie der modernen Gesellschaft. Band 4. Frankfurt a. M. Suhrkamp.

Luhmann, N. (2002) Die Religion der Gesellschaft. Hg. v. André Kieserling. Frankfurt a. M.: Suhrkamp.

Luhmann, N. (2005) Einführung in die Theorie der Gesellschaft. Hg. v. Dirk Baecker. Heidelberg: Auer.

Luhmann, N. (2009a) Haltlose Komplexität. In: Ders. Soziologische Aufklärung 5. Konstruktivistische Perspektiven. Wiesbaden: VS.

Luhmann, N. (2009b) Das Paradox der Menschenrechte und drei Formen seiner Entfaltung. In: Ders. Soziologische Aufklärung 6. Die Soziologie und der Mensch. Wiesbaden: VS.

Luhmann, N. (2009c) Reflexive Mechanismen. In: Ders. Soziologische Aufklärung 1. Aufsätze zur Theorie sozialer Systeme. Wiesbaden: VS.

Luhmann, N. (2009d) Gesellschaft. In: Ders. Soziologische Aufklärung 1. Aufsätze zur Theorie sozialer Systeme. Wiesbaden: VS.

Luhmann, N. (2009e) Interaktion, Organisation, Gesellschaft. In: Ders. Soziologische Aufklärung 2. Aufsätze zur Theorie der Gesellschaft. Wiesbaden: VS.

Luhmann, N. (2009f) Die Weltgesellschaft. In: Ders. Soziologische Aufklärung 2. Aufsätze zur Theorie der Gesellschaft. Wiesbaden: VS.

Luhmann, N. (2009g) Inklusion und Exklusion. In: Ders. Soziologische Aufklärung 6. Die Soziologie und der Mensch. Wiesbaden: VS.

Luhmann, N. (2010) Politische Soziologie. Hg. v. André Kieserling. Berlin: Suhrkamp.

Luhmann, N. (2011) Organisation und Entscheidung. Wiesbaden: VS.

Mayntz, R.; Rosewitz, B.; Schimank, U.; Stichweh, R. (1988) (eds.) Differenzierung und Verselbständigung. Zur Entwicklung gesellschaftlicher Teilsysteme. Frankfurt a. M.; New York: Campus. 
Musolff, A. (2011) Metaphorische Parasiten und „parasitäre“ Metaphern: Semantische Wechselwirkungen zwischen politischem und naturwissenschaftlichem Vokabular. In: Junge, M. (ed.) Metapher und Gesellschaft. Die Bedeutung der Orientierung durch Metaphern. Wiesbaden: VS.

Nassehi, A. (2004a) Eliten als Differenzierungsparasiten. Skizze eines Forschungsprogramms. In: Hitzler, R.; Hornborstel, S.; Mohr, C. (eds.) Elitenmacht. Wiesbaden: VS.

Nassehi, A. (2004b) Die Theorie funktionaler Differenzierung im Horizont ihrer Kritik. In: Zeitschrift für Soziologie 33(2): $98-118$.

Nesselrath, H.-G. (2000) „Parasit“. In: Der Neue Pauly. Band 9. Stuttgart: Metzler.

Ohlendieck, L. (2003) Gender Trouble in Organisationen und Netzwerken. In: Pasero, U.; Weinbach, C. (eds.) Frauen, Männer, Gender Trouble. Systemtheoretische Essays. Frankfurt a. M.: Suhrkamp.

Opitz, S. (2012) An der Grenze des Rechts. Inklusion/Exklusion im Zeichen der Sicherheit. Weilerswist: Velbrück.

Pernerstorfer, M. J. (2012) Prolegomena zu einer Kulturgeschichte des Parasiten in der griechisch-römischen Komödie. In: Literatur- und Theatersoziologie 7: 99-116.

Reckwitz, A. (2004) Die Logik der Grenzerhaltung und die Logik der Grenzüberschreitungen: Niklas Luhmann und die Kulturtheorien. In: Burkart, G.; Runkel, G. (eds.) Luhmann und die Kulturtheorie. Frankfurt a. M.: Suhrkamp.

Röttgers, K. (1999) Michel Serres. Strukturen mit Götterboten. In: Jurt, J. (ed.) Von Michel Serres bis Julia Kristeva. Freiburg: Rombach.

Schneider, W. L. (2008) Terrorismus und andere Parasiten. Ein systemtheoretischer Deutungsversuch der Intitialphase des nordirischen Konflikts. In: Bonacker, T.; Greshoff, R.; Schimank, U. (eds.) Sozialtheorien im Vergleich. Der Nordirlandkonflikt als Anwendungsfall. Wiesbaden: VS.

Schneider, W. L.; Kusche, I. (2010) Parasitäre Netzwerke in Wissenschaft und Politik. In: Bommes, M.; Tacke, V. (eds.) Netzwerke in der funktional differenzierten Gesellschaft. Wiesbaden: VS.

Schroer, M. (2010) Funktionale Differenzierung versus soziale Ungleichheit. Ein Beitrag zur Debatte über die Grundstruktur der modernen Gesellschaft. In: Kneer, G.; Moebius, S. (eds.) Soziologische Kontroversen. Beiträge zu einer anderen Geschichte der Wissenschaft vom Sozialen. Berlin: Suhrkamp.

Schwinn, T. (ed.) (2004) Differenzierung und soziale Ungleichheit. Die zwei Soziologien und ihre Verknüpfung. Frankfurt a. M.: Humanities Online.

Serres, M. (1987) Der Parasit. Frankfurt a. M.: Suhrkamp.

Stäheli, U. (1996) From Victimology towards Parasitology. A Systems Theoretical Reading of the Function of Exclusion. In: Recherches sociologiques 27(2): 59-80.

Stäheli, U. (2008) System: Unentscheidbarkeit und Differenz. In: Moebius, S.; Reckwitz, A. (eds.) Poststrukturalistische Sozialwissenschaften. Frankfurt a. M.: Suhrkamp 2008.

Stäheli, U. (2003) Sinnzusammenbrüche. Eine dekonstruktive Lektüre von Niklas Luhmanns Systemtheorie. Weilerswist: Velbrück. 
Stichweh, R. (1984) Zur Entstehung des modernen Systems wissenschaftlicher Disziplinen. Physik in Deutschland 1740-1890. Frankfurt a. M.: Suhrkamp.

Stichweh, R. (2000) Die Weltgesellschaft. Soziologische Analysen. Frankfurt a. M.: Suhrkamp. 